\title{
Fuzzy Support Vector Machines for Face Recognition: A Review
}

\author{
Navin Prakash \\ Research Scholar \\ IFTM University, Moradabad
}

\author{
Yashpal Singh, PhD \\ Associate Professor \\ B.I.E.T.-Jhansi
}

\begin{abstract}
Support vector machine (SVMs) is a classical classification tool in face recognition. In ordinary SVM, every input points are considered to have the same commitment to the training model. On the other hand, this is not generally valid due to some challenges in face recognition. Since there may be a few points undermined by commotion so they are less significant and the machine ought to better to toss them which are undecidable. This paper review some methodology to handle this sort of information giving so as to utilize fuzzy methodology them a weight which demonstrate the diverse commitment of every point to the model. The weights are resolved as for their membership function. Such approach is typically called as Fuzzy SVM (FSVM).
\end{abstract}

\section{Keywords}

Face Recognition, Support vector Machines, Fuzzy Support vector Machines.

\section{INTRODUCTION}

Fuzzy SVM was initially presented by Shigeo Abe in 2001 to determine the unclassifiable areas issue. At that point, in 2002, Fuzzy SVM utilized as a part of diverse routes by Chun-Fu Lin to diminish the impact of outliers in model generation. In routine SVM, the sample points are treated equally, including outlier and every point is thought to be fit in with one class. Be that as it may, in face classification, some input points may not precisely allocated to one of these classes due to various problems possess in face recognition and different points have the diverse commitment to the decision surface. So there are required to build a robust face recognition system.

In face recognition process, the impacts of the training points are distinct. Some training points are more critical than others in the classification issue. We would require that the important training points must be classified accurately .every training point not precisely fits in with one of the two classes. It might $90 \%$ have a place with one class and $10 \%$ be aimless, and other situation. So, there is a fuzzy membership $0<\mathrm{Q}_{i} \leq$ 1 connected with each training point $\boldsymbol{x}_{\boldsymbol{i}}$. This fuzzy membership can be viewed as the disposition of the relating training point toward one class in the classification issue and the value $\left(1-2_{i}\right)$ can be regarded as the attitude of meaningless. We augment the idea of SVM with fuzzy membership and make it a FSVM.

\section{LITERATURE REVIEW}

A few looks into have been directed in Fuzzy SVM.Chun-Fu Lin and Sheng-De Wang proposed Fuzzy SVM to take care of such issue by weighting the data training so that diverse input points can make distinctive commitments to the learning of choice
Surface [Chun-Fu Lin, 2002] as talked about in point of interest beneath. For non-successive dataset, they assess the distance

Between every data to its class center as a membership function. This technique is broadly acknowledged and utilized as a part of a few papers. Qin-Yu Ping utilized this system to tackle the multi-class issue [Qin-Yu Ping, 2008].Hao Tang likewise utilized such distance function joined with affinity variable to measure the input points [Hao Tang, 2008].Nonetheless, this technique dependably considers the outer data as outlier in light of the fact that they are given a very small membership value, about or nearly or exactly the same as lower bound value that has been indicated. Though, not all outer data can be considered as an outlier since they have comparative trademark with other data in the same class. In addition, they utilized Mean value as the center of the class, while it is simply influenced by the outlier. Some approaches given by the researchers given below.

\section{Face recognition in color image using PCA and FSVM (2005)}

This paper [1], is related to recognition of human face within color image. The proposed technique utilized the clue of skin color to identify faces and malate wavelet transform was applied to get LL2 frequency sub band picture of the original feces than feature extracted from the raw data using PCA. These discriminative and low dimensional feature achieved were fed to a SVM with polynomial kernel for the training and fuzzy theory was applied on the multi class classification to manage the unclassifiable cases. Examinations were led utilizing the indoors photo and the outcomes showed that the proposed strategy was productive for the frontal face detection and recognition. As per this paper polynomial kernel was at degree 2, SVM give the result 93.72 while fuzzy SVM given 96.22 at the degree 3 SVM given 95.64 and $97.74 \%$ by using FSVM.

Fuzzy Support Vector Machines (2005)

A SVM takes in the decision surface from two distinct classes of the input points. In so many applications, every input points may not be completely relegated to one of these two classes. In this paper, Researchers applied a fuzzy membership to every input point and reformulate the SVMs .because every input points can make different contributions to the learning of decision surface. They call the proposed technique fuzzy SVMs (FSVMs). In this paper [2], proposed the FSVM that forces a fuzzy membership to every input point to contribute different supports to the learning of decision surface.

Face Recognition Using Total Margin-Based Adaptive Fuzzy Support Vector Machines (2007)

This paper [3], proposed another classifier called total marginbased adaptive fuzzy support vector machines (TAF-SVM) that tackle some issues that fall in support vector machines (SVMs) related to the face recognition. The proposed TAFSVM not just tackle overfitting issue came because of the 
outlier with the method of fuzzification of the penalty, additionally amends the skew of the optimal separating hyperplane because of the imbalanced data by using distinct cost algorithm. Likewise, by acquainted the total margin algorithm with supplant the conventional soft-margin algorithm, a lower generalization error bound can be acquired. These three functions are embodied into the traditional SVM so that the TAF-SVM is proposed and reformulated in both linear and nonlinear cases, trial results demonstrate that the proposed TAF-SVM is better than SVM as far as the facerecognition accuracy. The result shows that the proposed TAF-SVM can achieve smaller error variances than SVM.

\section{Fuzzy Theory Based Support Vector Machine Classifier (2008)}

In This paper [4], researchers applied fuzzy theory to SVM. As fuzzy membership to every input data point of SVM can make distinctive contributions to the every class then, the SVM's kernel's parameters are calculated by the kernel's parameters evaluation function. Test results affirm the prevalence of the displayed technique over the customary SVM classifier.

In [5], when tackling Two-Class classification problem utilizing conventional SVM, every training point is dealt with similarly and doled out to one and stand out class. On the other hand, in numerous genuine word issues, there are cases that some training points are polluted by noise. Also, a few points in the training data are lost on the wrong side unintentionally. These points are all outliers; in this manner don't totally belong to one class, however with different memberships in the two classes. For this situation, the traditional SVM training algorithm will settle on the decision boundary to extremely go amiss from the optimal hyperplane in light of the fact that SVM is exceptionally sensitive to outliers [5, 6, and 7].To take care of this issue, a few methods have been overseen. In Ref. [34], a central SVM strategy is proposed to utilize the class centers in building the SVM.In Ref. [8], an adaptive margin SVM is created in light of the use of adaptive margins for every training pattern. In Ref. [36], the first input space is mapped to a normalized feature space to expand the strength to noise. In Ref. [5], a robust support vector machine is proposed going for taking care of the overfitting issue as Fuzzy support vector machine (FSVM).

As mention above that in [9], different kinds of research have been directed in Fuzzy SVM. Chun-Fu Lin and Sheng-De Wang proposed Fuzzy SVM to settle such issue by weighting the data training so that different input points can make different contributions to the learning of decision surface [Chun-Fu Lin, 2002]. For non-sequential dataset, they assess the separation between every data to its class center as membership function. This system is generally accepted and utilized.

In Reference [10], a standout amongst the most vital things to FSVM is picking fuzzy memberships for a given issue. In this paper proposed another fuzzy membership function for the nonlinear SVM.Researchers calculate fuzzy membership in the feature space and represent it with kernels. They proved that this system has great performance by reducing the effects of outliers and enhances the accuracy and generalization. SVM and FSVM can be utilized to take care of Two-Class issue and also Multi-Class issue. The classification issue said in this paper is bound to Two-Class Problem; out withstanding, the proposed technique in this paper can likewise be connected to Multi-Class issue.
In reference [11], Hao Tang utilized such distance function combined with affinity variable to measure the input points. Nonetheless, this strategy dependably considers the outer data as the outlier in light of the fact that they are given a small membership value, almost or the very same as the lower bound value that has been determined. While, not all outer data can be considered as an outlier since they have comparative trademark with other data in the same class. Additionally, they utilized Mean value as the center of the class while it is simply influenced by the outlier.

In this paper [12], researchers presented fuzzy support vector machine (FSVM) and define a membership function to classify pictures which are unclassifiable utilizing ordinary SVM.For the input vector of SVM and FSVM, they utilize combined image feature histogram. Being compared and the conventional SVM, FSVM demonstrates the same result as SVM for the pictures in the classifiable regions, what's more, for those in the unclassifiable regions, FSVM produces preferred result over SVM.

This paper [13], Support vector machine (SVM) is a hypothetically all around persuaded calculation created from statistical learning theory that have indicated great performance in numerous fields. Regardless of its prosperity, despite everything it experiences a noise sensitivity issue. To unwind this issue, the SVM was stretched out by the acquaintance of fuzzy memberships with the fuzzy SVM (FSVM).The FSVM additionally has been amplified further in two courses: by embracing a changed objective function with the help of domain-specific information and by utilizing an alternate membership calculation technique. In this paper, they proposed another membership calculation strategy that fits in with the second group. It is unique in relation to past ones in that it doesn't expect any simple data distribution and does not require any former knowledge. The proposed strategy depends on reconstruction error, which measures the agreement between the overall data structure and a data point. In this way, the reconstruction error can speak to the degree of outlier-ness and help in accomplishing noise robustness. Test results with synthetic and real data set also support this.

In this reference [14] (2013), as SVM is sensitive to outliers or noises in the dataset, Fuzzy SVM (FSVM) used. Like SVM, despite everything it goes for discovering an optimal hyperplane that can isolate two classes with the maximal margin. The main distinction is that fuzzy membership is appointed to training point, which makes it sensitive to outliers or noises. They proposed another membership function within-class scatter WCS in FSVM. The WCSFSVM not only enhance the accuracy and generalization also handle the classification problems with outliers or noises with more adequately which occur in SVM.

In reference [15] (2015), the issue of one-against-all support vector machines (SVMs) for multilabel classifications that an data sample may be classified into a multilabel class that is not defined or it may not be classified into any class. To take care of this issue, in this paper authors propose fuzzy SVMs (FSVMs) for multilabel classification, in which for each multilabel class, a region with the related membership function is characterized and data point is classified into a multilabel class whose membership function is the biggest. By computer tests, they demonstrated that the accuracy is enhanced by the FSVM over the ordinary one-against-all SVM. 


\section{ANALYSIS OF THE RESEARCH RESULTS}

In face recognition systems faces various challenges all method wants to be robust in all situation. Therefore in classification step every input data point of SVM can make distinctive contributions to the every class. All researchers redesign the SVM as FSVM by assigning a membership function to every inputs, because SVM is sensitive against outliers and noise. Therefore choosing a proper fuzzy membership function, a FSVM can successfully reduce the effects of outliers in face classification problem. Chun-Fu Lin and Sheng-De Wang proposed Fuzzy SVM they uses the distance between every data to its class center for a membership function. TAF-SVM handle overfitting issue came from outlier. Reference [4], calculated by the kernel's parameters evaluation function. Hao Tang utilized such distance function combined with affinity variable to measure the input points. Another FSVM, they utilize combined image feature histogram. On the other hand all researchers are modifying a suitable membership function in face recognition for achieving the good results.

\section{CONCLUSIONS \& FUTURE DIRECTIONS}

Face recognition is an inspiring problem in the field of Image analysis and facing various challenges such as facial expression, perspiration, hair styles, cosmetics, and changes due to aging so numerous problem occurs in face classification. For face classification, Specialists show the prevalence of the FSVM over the SVM because SVM is sensitive to outliers or noises so FSVM yields wished result over SVM by using fuzzy data which is unclassifiable. But there stays some future work as, automatically determine a suitable fuzzy membership work that can diminish the impact of noises and outliers for a class of issues

\section{ACKNOWLEDGMENTS}

Sincere thanks to my Ph.D. supervisor Dr.Yashpal Singh, Associate Professor at BIET-Jhansi, India, who encourage me and friendly advice. The authors sincerely thank to the Dr. R.M. Dubey, Vice-Chancellor, IFTM University, Moradabad \& Prof. MIH Ansari, Director, SET-IFTM University, Moradabad for his unstinted support and motivation.

\section{REFERENCES}

[1] Xutao Zhang; Yudong Guan; Shen Wang; Jianquan Liang; Taifan Quan;" Face recognition in color images using principal component analysis and fuzzy support vector machines " 1st International Symposium on Systems and Control in Aerospace and Astronautics, ISSCAA, 2006.4 pp. - 45,2006.

[2] Sheng-Wu Xiong; Hong-Bing Liu; Xiao-Xiao Niu; "Fuzzy support vector machines based on FCM clustering "Proceedings of 2005 International Conference on Machine Learning and Cybernetics, Vol. 5, pp: $2608-2613,2005$.
[3] Yi-Hung Liu; Yen-Ting Chen; "Face Recognition Using Total Margin-Based Adaptive Fuzzy Support Vector Machines "IEEE Transactions on Neural Networks, Volume: 18 , Issue: 1, pp: 178 - 192, 2007.

[4] Xuehua Li; Lan Shu; "Fuzzy Theory Based Support Vector Machine Classifier "Fifth International Conference on Fuzzy Systems and Knowledge Discovery, 2008. FSKD '08. Volume: 1,pp: $600-$ 604,2008 .

[5] Song Q Robust support vector machine with bullet hole image classification. IEEE Trans Syst Cybern 32(4):440 448, 2002.

[6] Guyon I, Matic N, Vapnik VN (1996) Discovering information patterns and data cleaning. MIT Press, Cambridge, pp 181-203, 1996.

[7] P. J. Phillips "Support Vector Machines Applied to Face Recognition", pp. 803-809, Advances in Neural Information Processing Systems 11, MIT Press, 1999.

[8] Herbrich R, Weston J," Adaptive margin support vector machines for classification" In: Proceedings of the 9th ICANN,vol 2, Sept 1999, pp. 880 C 885. 649-668, 2001.

[9] Chun-Fu Lin, S.-D. W. ," Fuzzy Support Vector Machines". IEEE Transactions on Neural Networks, 2002

[10] Xiufeng Jiang $Æ$ Zhang Yi $Æ$ Jian Cheng Lv, "Fuzzy SVM with a new fuzzy membership function" Neural Comput \& Applic 15,pp: 268-276.

[11] H. Tang and L.-S. Qu, "Fuzzy support vector machines with a new fuzzy membership function for pattern classification", Proc. 7th Int. Conf. Mach. Learning Cybern., pp.768 -773, 2008.

[12] Jianming Li ; , Dalian ; Shuguang Huang ; Rongsheng $\mathrm{He}$; Kunming Qian, "Image Classification Based on Fuzzy Support Vector Machine Computational Intelligence and Design", ISCID '08. IEEE International Symposium on (Volume:1)Page(s):68-71,2008.

[13] Gyeongyong Heo, Gainesville, FL, USA ; Gader, P., "Fuzzy SVM for noisy data: A robust membership calculation method, Fuzzy Systems", FUZZ-IEEE 2009. IEEE International Conference on, Jeju Island, Page(s):431 - 436, 2009.

[14] Wenjuan An, Mangui Liang,"Fuzzy support vector machine based on within-class scatter for classification problems with outliers or noises", Original Research Article Neurocomputing, Volume 110, Pages 101-110 .2013

[15] Shigeo Abe,"Fuzzy support vector machines for multilabel classification" Original Research Article Pattern Recognition, Volume 48, Issue 6, Pages 21102117,2015 